\title{
BOUNDS ON THE LATTICE RULE CRITERION $R$
}

\author{
STEPHEN JOE
}

\begin{abstract}
Lattice rules are used for the numerical integration of periodic functions over the $s$-dimensional unit cube. They are normally classified according to their 'rank'; in simple terms, the rank of a lattice rule is the minimum number of sums required to write it down. One criterion for measuring the 'goodness' of a lattice rule is the quantity $R$ which is the quadrature error for a certain test function. Bounds on $R$ exist for rank-1 and rank-2 lattice rules, but not for lattice rules of higher rank. For $1 \leq m \leq s$, we shall look at certain rank- $m$ rules and obtain bounds on $R$ for them. These rank- $m$ rules have $n^{m} r$ quadrature points, where $n$ and $r$ are relatively prime numbers. In order to obtain these bounds, we make use of a result which shows that $R$ may be considered to be the quadrature error obtained when a modified lattice rule with only $r$ quadrature points is applied to a modified test function. Some numerical results are given.
\end{abstract}

\section{INTRODUCTION}

Lattice rules are equal-weight quadrature rules used in the numerical evaluation of integrals of the form

$$
I f=\int_{U^{s}} f(\mathbf{x}) d \mathbf{x},
$$

where

$$
U^{s}=\left\{\mathbf{x} \in \mathbb{R}^{s}: 0 \leq x_{k}<1,1 \leq k \leq s\right\}
$$

is the half-open unit cube in $s$ dimensions. The function $f$ is assumed to be 1-periodic in each of its $s$ variables. The abscissa set $\left\{\mathbf{x}_{0}, \ldots, \mathbf{x}_{N-1}\right\}$ of the lattice rule

$$
Q f=\frac{1}{N} \sum_{j=0}^{N-1} f\left(\mathbf{x}_{j}\right)
$$

consists of all the points in some 'integration lattice' that also belong to $U^{s}$. A lattice is a discrete set of points in $\mathbb{R}^{s}$ such that the sum and difference of every point in the set also belongs to the set; it is an integration lattice if it contains the integer lattice $\mathbb{Z}^{s}$ as a sublattice. A lattice rule with $N$ distinct abscissae is said to be of order $N$.

Received by the editor May 18, 1992.

1991 Mathematics Subject Classification. Primary 65D30, 65D32.

The continuing financial support of the Australian Research Council is gratefully acknowledged.

(C) 1993 American Mathematical Society $0025-5718 / 93 \$ 1.00+\$ .25$ per page 
A classification of lattice rules based on the concept of 'rank' may be found in [10]. There we find the result that every lattice rule may be written as an expression of the form

$$
Q f=\frac{1}{N} \sum_{j_{m}=0}^{n_{m}-1} \cdots \sum_{j_{1}=0}^{n_{1}-1} f\left(\frac{j_{1}}{n_{1}} \mathbf{z}_{1}+\cdots+\frac{j_{m}}{n_{m}} \mathbf{z}_{m}\right),
$$

where $\mathbf{z}_{1}, \ldots, \mathbf{z}_{m}$ are integer vectors, $m$, which satisfies $1 \leq m \leq s$, is the rank of the rule, $n_{k+1}$ divides $n_{k}$ for $k=1, \ldots, m-1$, and $n_{m} \geq 2$. The order of the rule is given by $N=n_{1} n_{2} \cdots n_{m}$. The rank and the invariants $n_{1}, \ldots, n_{m}$ are uniquely determined numbers. (The abscissae as they appear in (1.2) may not lie in $U^{s}$, but abscissae that do lie in $U^{s}$ may be obtained by subtraction of appropriate integer vectors; the assumed periodicity of $f$ ensures that this subtraction leaves the lattice rule unchanged.)

An important example of a lattice rule is the method of good lattice points due to Korobov [6] and Hlawka [2], in which the rule is of the rank-1 form

$$
Q f=\frac{1}{N} \sum_{j=0}^{N-1} f\left(\frac{j}{N} \mathbf{z}\right) \text {. }
$$

Here $\mathbf{z}$ is an integer vector of length $s$ having no nontrivial factor common with $N$.

The error in the lattice rule $Q$ is easily stated.

Theorem 1 [9]. Suppose $Q$ is the lattice rule (1.1) and $f$ has the absolutely convergent Fourier series representation

$$
f(\mathbf{x})=\sum_{\mathbf{h} \in \mathbb{Z}^{s}} a(\mathbf{h}) e^{i 2 \pi \mathbf{h} \cdot \mathbf{x}} .
$$

Then

$$
Q f-I f=\sum_{\mathbf{h} \in L^{\perp}}^{\prime} a(\mathbf{h}) .
$$

In the theorem, $\mathbf{h} \cdot \mathbf{x}$ is the usual inner product in $s$ dimensions, the prime on the sum indicates that the $\mathbf{h}=\mathbf{0}$ term is omitted, and $L^{\perp}$ is the 'dual lattice' defined by

$$
L^{\perp}:=\left\{\mathbf{h} \in \mathbb{Z}^{s}: \mathbf{h} \cdot \mathbf{x} \in \mathbb{Z} \forall \mathbf{x} \in L\right\} ;
$$

it is the dual of the lattice $L(Q)$ which corresponds to $Q$.

There are several criteria available for measuring the 'goodness' of a lattice rule, all coming from the number-theoretic literature associated with the method of good lattice points. One such criterion is given by the quantity $R$ (see [7] and [8]), which for a lattice rule of order $N$ is defined by

$$
R=R(Q):=\sum_{\mathbf{h} \in L^{\perp} \cap \mathbf{E}(N)}^{\prime} \frac{1}{\bar{h}_{1} \bar{h}_{2} \cdots \bar{h}_{s}},
$$

where $\bar{h}=\max (1,|h|)$, and $\mathbf{E}(N)=\left\{\mathbf{h} \in \mathbb{Z}^{s}:-N / 2<h_{k} \leq N / 2,1 \leq k \leq s\right\}$. If

$$
f_{N}(\mathbf{x})=\sum_{\mathbf{h} \in \mathbf{E}(N)} \frac{e^{i 2 \pi \mathbf{h} \cdot \mathbf{x}}}{\bar{h}_{1} \bar{h}_{2} \cdots \bar{h}_{s}}=\prod_{k=1}^{s} F_{N}\left(x_{k}\right),
$$


where

$$
F_{N}(x)=\sum_{-N / 2<h \leq N / 2} \frac{e^{i 2 \pi h x}}{\bar{h}}=1+\sum_{h \in E^{*}(N)} \frac{e^{i 2 \pi h x}}{|h|},
$$

with $E^{*}(N):=\{h \in \mathbb{Z}:-N / 2<h \leq N / 2, h \neq 0\}$, then we see from (1.4) that

$$
R(Q)=Q f_{N}-I f_{N}=Q f_{N}-1
$$

In Joe and Sloan [5] an asymptotic series was used to approximate the function $F_{N}(x)$ for $x$ sufficiently far away from 0 , allowing $R$ to be calculated, using (1.5) and (1.7), in $O(N)$ operations.

Currently, there exist bounds on $R$ for only rank-1 (see [7]) and rank-2 (see [8]) lattice rules. These latter bounds do not have explicit constants. In this paper, we shall provide some new bounds on $R$ (with explicit constants) for the lattice rules given by

$$
Q_{m} f=\frac{1}{n^{m} r} \sum_{k_{m}=0}^{n-1} \ldots \sum_{k_{1}=0}^{n-1} \sum_{j=0}^{r-1} f\left(\frac{j}{r} \mathbf{z}+\frac{\left(k_{1}, \ldots, k_{m}, 0, \ldots, 0\right)}{n}\right),
$$

where $m=0,1, \ldots, s, \mathbf{z}$ and $r$ have no nontrivial common factor, and $n$ and $r$ are relatively prime. Such lattice rules arise from the imbedded sequences of lattice rules found in [4]. From there we have the result that for $1 \leq m \leq s$, $Q_{m}$ is a lattice rule of rank $m$ while $Q_{0}$ is evidently the rank-1 rule given by (1.3). Thus, here we obtain bounds on $R$ for lattice rules having ranks from 1 to $s$ inclusive. For simplicity, we shall only consider the case when $r$ is prime. The bounds that are to be found in $\S 3$ are obtained by deriving an expression for a certain mean value of $R\left(Q_{m}\right)$.

The derivation of this expression for the mean makes use of a result which shows that $R\left(Q_{m}\right)$ may be considered to be the quadrature error obtained when a certain $r$-point rank-1 rule is applied to a modified function. This result is given in $\S 2$. Besides being of theoretical use here, this result is also of practical use, since it enables $R\left(Q_{m}\right)$ to be calculated more efficiently (recall that the lattice rule $Q_{m}$ has $n^{m} r$ quadrature points).

Section 4 concludes the paper with some numerical results.

\section{A PRELIMINARY RESULT}

Here we consider the rank- $m$ lattice rules given by $(1.8)$, that is,

$$
Q_{m} f=\frac{1}{n^{m} r} \sum_{k_{m}=0}^{n-1} \ldots \sum_{k_{1}=0}^{n-1} \sum_{j=0}^{r-1} f\left(\frac{j}{r} \mathbf{z}+\frac{\left(k_{1}, \ldots, k_{m}, 0, \ldots, 0\right)}{n}\right),
$$

where $m=1, \ldots, s, \mathbf{z}$ and $r$ have no nontrivial common factor, and $n$ and $r$ are relatively prime. Though $Q_{m}$ has order $n^{m} r$, we now show how it is possible to obtain $R\left(Q_{m}\right)$ by using only $r$ evaluations of a modified function $f_{N}^{(n)}$, rather than $n^{m} r$ evaluations of $f_{N}$ (with $N=n^{m} r$ ). This result, modeled on an analogous result in [4], will be used in the next section to obtain bounds on $R\left(Q_{m}\right)$. 
Theorem 2. For $1 \leq m \leq s$ and $n \geq 2$, let $f_{N}^{(n)}$ be the function on $\mathbb{R}^{s}$ defined by

$$
f_{N}^{(n)}(\mathbf{x}):=\left(\prod_{\ell=1}^{m} F_{N}^{(n)}\left(x_{\ell}\right)\right) \prod_{p=m+1}^{s} F_{N}\left(x_{p}\right)
$$

where

$$
F_{N}^{(n)}(x)=1+\frac{1}{n} \sum_{h \in E^{*}(N / n)} \frac{e^{l 2 \pi h x}}{|h|},
$$

and define the r-point rank-1 rule by

$$
Q_{m}^{(n)} f:=\frac{1}{r} \sum_{j=0}^{r-1} f\left(\frac{j n}{r} z_{1}, \ldots, \frac{j n}{r} z_{m}, \frac{j}{r} z_{m+1}, \ldots, \frac{j}{r} z_{s}\right),
$$

where $\mathrm{z}=\left(z_{1}, \ldots, z_{s}\right)$. Then

$$
R\left(Q_{m}\right)=Q_{m}^{(n)} f_{N}^{(n)}-1 .
$$

Proof. Using (1.7) and (1.8), we obtain

$$
\begin{aligned}
R\left(Q_{m}\right)= & \frac{1}{n^{m} r} \sum_{k_{m}=0}^{n-1} \cdots \sum_{k_{1}=0}^{n-1} \sum_{j=0}^{r-1} f_{N}\left(\frac{j}{r} \mathbf{z}+\frac{\left(k_{1}, \ldots, k_{m}, 0, \ldots, 0\right)}{n}\right)-1 \\
= & \frac{1}{n^{m} r} \sum_{k_{m}=0}^{n-1} \cdots \sum_{k_{1}=0}^{n-1} \sum_{j=0}^{r-1}\left(\prod_{\ell=1}^{m} \sum_{-N / 2<h \leq N / 2} \frac{e^{i 2 \pi h\left(j z_{\ell} / r+k_{\ell} / n\right)}}{\bar{h}}\right) \\
& \times \prod_{p=m+1}^{s} F_{N}\left(\frac{j}{r} z_{p}\right)-1 \\
= & \frac{1}{r} \sum_{j=0}^{r-1} K_{j}^{(n, m)} \prod_{p=m+1}^{s} F_{N}\left(\frac{j}{r} z_{p}\right)-1,
\end{aligned}
$$

where we have used (1.5) and (1.6) in the second step, and

$$
\begin{aligned}
K_{j}^{(n, m)} & =\frac{1}{n^{m}} \sum_{k_{m}=0}^{n-1} \cdots \sum_{k_{1}=0}^{n-1} \prod_{\ell=1}^{m}\left(1+\sum_{h \in E^{*}(N)} \frac{e^{i 2 \pi h\left(j z_{\ell} / r+k_{\ell} / n\right)}}{|h|}\right) \\
& =\prod_{\ell=1}^{m} \frac{1}{n} \sum_{k=0}^{n-1}\left(1+\sum_{h \in E^{*}(N)} \frac{e^{i 2 \pi h j z_{\ell} / r}}{|h|}\left(e^{i 2 \pi h / n}\right)^{k}\right) \\
& =\prod_{\ell=1}^{m} \frac{1}{n}\left(n+\sum_{\substack{h \in E^{*}(N) \\
h \equiv 0(\bmod n)}} \frac{e^{i 2 \pi h j z_{\ell} / r}}{|h|} n\right)=\prod_{\ell=1}^{m}\left(1+\sum_{n h \in E^{*}(N)} \frac{e^{i 2 \pi n h j z_{\ell} / r}}{|n h|}\right) \\
& =\prod_{\ell=1}^{m}\left(1+\frac{1}{n} \sum_{h \in E^{*}(N / n)} \frac{e^{i 2 \pi h j n z_{\ell} / r}}{|h|}\right)=\prod_{\ell=1}^{m} F_{N}^{(n)}\left(\frac{j n}{r} z_{\ell}\right) .
\end{aligned}
$$


Thus,

$$
R\left(Q_{m}\right)=\frac{1}{r} \sum_{j=0}^{r-1}\left(\prod_{\ell=1}^{m} F_{N}^{(n)}\left(\frac{j n}{r} z_{\ell}\right)\right) \prod_{p=m+1}^{s} F_{N}\left(\frac{j}{r} z_{p}\right)-1,
$$

which is equivalent to $(2.4)$.

To apply this theorem in practice, we need to be able to evaluate the functions $F_{N}^{(n)}$ given by (2.2). However, from (1.6) and (2.2) we have

$$
F_{N}^{(n)}(x)=\frac{1}{n} F_{N / n}(x)+1-\frac{1}{n}
$$

Thus, $F_{N}^{(n)}$ is no more difficult to evaluate than $F_{N}$. In fact, since $F_{N / n}=F_{n^{m-1} r}$ has less terms than $F_{N}=F_{n^{m} r}$, evaluation of $F_{N}^{(n)}$ may well require less work than evaluation of $F_{N}$.

\section{Bounds on $R\left(Q_{m}\right)$}

As indicated in $\S 1$, we shall obtain bounds on $R\left(Q_{m}\right)$ for the case in which $r$ is prime by obtaining an expression for a certain mean value of $R\left(Q_{m}\right)$. The mean is taken over all integer vectors $\mathbf{z}$ for which $-r / 2<z_{k} \leq r / 2, z_{k} \neq 0$, $1 \leq k \leq s$; reasons for choosing these vectors $\mathbf{z}$ as the admissible vectors may be found in [1]. We denote the set of these $(r-1)^{s}$ vectors by $Z_{r}$. Thus, the mean $M_{m, r}^{(n)}$ is defined by

$$
M_{m, r}^{(n)}:=\frac{1}{(r-1)^{s}} \sum_{\mathbf{z} \in Z_{r}} R\left(Q_{m}\right)
$$

An expression for $M_{m, r}^{(n)}$ when $r$ is prime is given in the following theorem. We remark that it would be possible to obtain an expression for the mean of $R\left(Q_{m}\right)$ over appropriate admissible vectors $\mathrm{z}$ when $r$ is not prime. However, the expression would be much more difficult to obtain (compare [8], which derives an expression for the mean value of $R$ for rank-2 rules). We recall that $E^{*}(k)$ is defined for any positive integer $k$ by $E^{*}(k):=\{h \in \mathbb{Z}:-k / 2<h \leq$ $k / 2, h \neq 0\}$.

Theorem 3. For $1 \leq m \leq s, n \geq 2$, and $r$ a prime number so that $r$ does not divide $n$, we have

$$
\begin{aligned}
M_{m, r}^{(n)}= & \frac{1}{r}\left(1+\frac{S_{n^{m-1} r}}{n}\right)^{m}\left(1+S_{n^{m} r}\right)^{s-m} \\
& +\frac{r-1}{r}\left(1-\frac{S_{n^{m-1} r}-S_{n^{m-1}}}{n(r-1)}\right)^{m}\left(1-\frac{S_{n^{m} r}-S_{n^{m}}}{r-1}\right)^{s-m}-1,
\end{aligned}
$$

where, for any positive integer $k$,

$$
S_{k}=\sum_{h \in E^{*}(k)} \frac{1}{|h|}
$$

Proof. Let $\mathbf{g}$ be an integer vector of the form

$$
\mathbf{g}=\left(n z_{1}, \ldots, n z_{m}, z_{m+1}, \ldots, z_{s}\right),
$$


where $\mathbf{z}=\left(z_{1}, \ldots, z_{s}\right)$. Then, using (2.3) and (2.4), we have

$$
R\left(Q_{m}\right)=\frac{1}{r} f_{N}^{(n)}(\mathbf{0})+\frac{1}{r} \sum_{j=1}^{r-1} f_{N}^{(n)}\left(\frac{j}{r} \mathbf{g}\right)-1,
$$

where the $j=0$ term has been separated out because it is independent of $\mathbf{z}$. Since $N=n^{m} r$, it follows from (1.6), (2.1), and (2.2) that

$$
\begin{aligned}
f_{N}^{(n)}(\mathbf{0}) & =\left(\prod_{\ell=1}^{m}\left(1+\frac{1}{n} \sum_{h \in E^{*}\left(n^{m-1} r\right)} \frac{1}{|h|}\right)\right) \prod_{p=m+1}^{s}\left(1+\sum_{h \in E^{*}\left(n^{m} r\right)} \frac{1}{|h|}\right) \\
& =\left(1+\frac{S_{n^{m-1} r}}{n}\right)^{m}\left(1+S_{n^{m} r}\right)^{s-m} .
\end{aligned}
$$

Writing

$$
H_{m, r}^{(n)}=\frac{1}{r}\left(1+\frac{S_{n^{m-1}}}{n}\right)^{m}\left(1+S_{n^{m} r}\right)^{s-m},
$$

we then have

$$
R\left(Q_{m}\right)=H_{m, r}^{(n)}+\frac{1}{r} \sum_{j=1}^{r-1} f_{N}^{(n)}\left(\frac{j}{r} \mathbf{g}\right)-1 .
$$

Now let $G_{m}$ be the set of all integer vectors $\mathbf{g}$ of the form given above with $\mathbf{z} \in Z_{r}$. Then from the definition of $M_{m, r}^{(n)}$ in (3.1) we have

$$
\begin{aligned}
M_{m, r}^{(n)} & =\frac{1}{(r-1)^{s}} \sum_{\mathbf{g} \in G_{m}}\left[H_{m, r}^{(n)}+\frac{1}{r} \sum_{j=1}^{r-1} f_{N}^{(n)}\left(\frac{j}{r} \mathbf{g}\right)-1\right] \\
& =H_{m, r}^{(n)}+\frac{1}{r(r-1)^{s}} \sum_{\mathbf{g} \in G_{m}} \sum_{j=1}^{r-1} f_{N}^{(n)}\left(\frac{j}{r} \mathbf{g}\right)-1 .
\end{aligned}
$$

We shall write this as

$$
M_{m, r}^{(n)}=H_{m, r}^{(n)}+\theta_{m, r}^{(n)} .
$$

With $N=n^{m} r$, we see from (1.6), (2.1), and (2.2) and the definition of $G_{m}$ that

$$
\begin{aligned}
\theta_{m, r}^{(n)}= & \frac{1}{r(r-1)^{s}} \sum_{j=1}^{r-1} \sum_{z_{1} \in E^{*}(r)} \ldots \sum_{z_{s} \in E^{*}(r)}\left(\prod_{\ell=1}^{m}\left(1+\frac{1}{n} \sum_{h \in E^{*}\left(n^{m-1} r\right)} \frac{e^{i 2 \pi h j n z_{\ell} / r}}{|h|}\right)\right) \\
& \times \prod_{p=m+1}^{s}\left(1+\sum_{h \in E^{*}\left(n^{m} r\right)} \frac{e^{22 \pi h j z_{p} / r}}{|h|}\right)-1 \\
= & \frac{1}{r} \sum_{j=1}^{r-1}\left(\prod_{\ell=1}^{m} \frac{1}{r-1} \sum_{z_{\ell} \in E^{*}(r)}\left(1+\frac{1}{n} \sum_{h \in E^{*}\left(n^{m-1} r\right)} \frac{e^{l 2 \pi h j n z_{\ell} / r}}{|h|}\right)\right) \\
& \times \prod_{p=m+1}^{s} \frac{1}{r-1} \sum_{z_{p} \in E^{*}(r)}\left(1+\sum_{h \in E^{*}\left(n^{m} r\right)} \frac{e^{l 2 \pi h j z_{p} / r}}{|h|}\right)-1
\end{aligned}
$$




$$
\begin{aligned}
=\frac{1}{r} & \sum_{j=1}^{r-1}\left(1+\frac{1}{r-1} \sum_{z \in E^{*}(r)} \sum_{h \in E^{*}\left(n^{m-1} r\right)} \frac{e^{i 2 \pi h j n z / r}}{|n h|}\right)^{m} \\
& \times\left(1+\frac{1}{r-1} \sum_{z \in E^{*}(r)} \sum_{h \in E^{*}\left(n^{m} r\right)} \frac{e^{i 2 \pi h j z / r}}{|h|}\right)^{s-m}-1 .
\end{aligned}
$$

Thus, we can write

$$
\theta_{m, r}^{(n)}=\frac{1}{r} \sum_{j=1}^{r-1}\left(1+\frac{1}{r-1} T_{m, r}^{(n)}(j)\right)^{m}\left(1+\frac{1}{r-1} T_{m, r}^{(1)}(j)\right)^{s-m}-1,
$$

where, for $k=1$ and $k=n$, we define

$$
T_{m, r}^{(k)}(j):=\sum_{z \in E^{*}(r)} \sum_{h \in E^{*}\left(n^{m} r / k\right)} \frac{e^{i 2 \pi h j k z / r}}{|k h|}, \quad 1 \leq j \leq r-1 .
$$

Now separating out the terms with $h \equiv 0(\bmod r)$, we obtain

$$
\begin{aligned}
T_{m, r}^{(n)}(j) & =\sum_{z \in E^{*}(r)} \sum_{r h \in E^{*}\left(n^{m-1} r\right)} \frac{1}{|n r h|}+\sum_{z \in E^{*}(r)} \sum_{\substack{h \in E^{*}\left(n^{m-1} r\right) \\
h \neq 0(\bmod r)}} \frac{e^{i 2 \pi h j n z / r}}{|n h|} \\
& =(r-1) \sum_{h \in E^{*}\left(n^{m-1}\right)} \frac{1}{|n r h|}+\sum_{\substack { z \in E^{*}(r) \\
\begin{subarray}{c}{h \in E^{*}\left(n^{m-1} r\right) \\
h \neq 0(\bmod r){ z \in E ^ { * } ( r ) \\
\begin{subarray} { c } { h \in E ^ { * } ( n ^ { m - 1 } r ) \\
h \neq 0 ( \operatorname { m o d } r ) } }\end{subarray}} \frac{e^{i 2 \pi h j n z / r}}{|n h|} \\
& =(r-1) \frac{S_{n^{m-1}}}{n r}+\sum_{z \in E^{*}(r)} \sum_{\substack{h \in E^{*}\left(n^{m-1} r\right) \\
h \neq 0(\bmod r)}} \frac{e^{i 2 \pi h j n z / r}}{|n h|} .
\end{aligned}
$$

But for $h \not \equiv 0(\bmod r)$, we have

$$
\sum_{z \in E^{*}(r)} e^{i 2 \pi h j n z / r}=\sum_{-r / 2<z \leq r / 2} e^{i 2 \pi h j n z / r}-1=-1,
$$

since $r$ is prime (by assumption), and therefore $h j n \not \equiv 0(\bmod r)$ for $1 \leq j \leq$ $r-1$. Thus, we obtain

$$
\begin{aligned}
\frac{1}{r-1} T_{m, r}^{(n)}(j) & =\frac{S_{n^{m-1}}}{n r}+\frac{1}{r-1} \sum_{\substack{h \in E^{*}\left(n^{m-1} r\right) \\
h \neq 0(\bmod r)}} \frac{-1}{|n h|} \\
& =\frac{S_{n^{m-1}}}{n r}-\frac{1}{(r-1) n}\left(\sum_{h \in E^{*}\left(n^{m-1} r\right)} \frac{1}{|h|}-\sum_{r h \in E^{*}\left(n^{m-1} r\right)} \frac{1}{|r h|}\right) \\
& =\frac{S_{n^{m-1}}}{n r}-\frac{1}{(r-1) n}\left(S_{n^{m-1} r}-\frac{1}{r} \sum_{h \in E^{*}\left(n^{m-1}\right)} \frac{1}{|h|}\right) \\
& =\frac{S_{n^{m-1}}}{n r}-\frac{1}{(r-1) n}\left(S_{n^{m-1} r}-\frac{S_{n^{m-1}}}{r}\right) \\
& =-\frac{S_{n^{m-1} r}-S_{n^{m-1}}}{n(r-1)} .
\end{aligned}
$$


In an analogous manner, it can be shown that

$$
\frac{1}{r-1} T_{m, r}^{(1)}(j)=-\frac{S_{n^{m} r}-S_{n^{m}}}{(r-1)} .
$$

Since $T_{m, r}^{(n)}(j)$ and $T_{m, r}^{(1)}(j), 1 \leq j \leq r-1$, are independent of $j$, we see from (3.2) that (3.3) and (3.4) yield the desired result.

Bounds on $R$ follow from the obvious fact that there must be a $\mathbf{z} \in Z_{r}$ for which $R\left(Q_{m}\right) \leq M_{m, r}^{(n)}$. We thus obtain the following corollary.

Corollary 4. For $1 \leq m \leq s, n \geq 2$, and $r$ a prime number so that $r$ does not divide $n$, there exists a $\mathbf{z} \in Z_{r}$ such that

$$
\begin{aligned}
R\left(Q_{m}\right) \leq & \frac{1}{r} \\
& \left(1+\frac{S_{n^{m-1} r}}{n}\right)^{m}\left(1+S_{n^{m} r}\right)^{s-m} \\
& +\frac{r-1}{r}\left(1-\frac{S_{n^{m-1} r}-S_{n^{m-1}}}{n(r-1)}\right)^{m}\left(1-\frac{S_{n^{m} r}-S_{n^{m}}}{r-1}\right)^{s-m}-1 .
\end{aligned}
$$

Remark. Theorem 3 and Corollary 4 also hold for $m=0$. In that case, the right-hand side of (1.8) becomes the rank-1 rule (1.3) (with $N=r$ ). Thus, $M_{m, r}^{(n)}$ becomes the mean of $R$ for rank-1 rules of prime order $N$. Since $S_{n^{m}}=S_{1}=0$, the mean of $R$ for rank-1 rules of prime order $N$ may then be written as

$$
M_{0, N}=\frac{1}{N}\left(1+S_{N}\right)^{s}+\frac{N-1}{N}\left(1-\frac{S_{N}}{N-1}\right)^{s}-1 .
$$

Now it follows from [7, Lemmas 1 and 2] that

$$
S_{k}<2 \log (k)+\beta+\frac{1}{k^{2}},
$$

where $\beta=2 \gamma-\log (4) \approx-0.2319$. Since $S_{N}$ has $N-1$ terms, we have $0 \leq S_{N} \leq N-1$, and hence $0 \leq 1-S_{N} /(N-1) \leq 1$. By using (3.6) and then (3.7), we obtain

$$
M_{0, N}<\frac{1}{N}\left(1+S_{N}\right)^{s}<\frac{1}{N}\left(1+2 \log (N)+\beta+\frac{1}{N^{2}}\right)^{s}<\frac{1}{N}(0.81+2 \log (N))^{s}
$$

for $N \geq 5, N$ prime. Simple calculations show that this last bound also holds when $N=2$ and $N=3$. Thus, there exists a $\mathbf{z} \in Z_{N}$ such that

$$
R\left(Q_{0}\right)<\frac{1}{N}(0.81+2 \log (N))^{s},
$$

which recovers the result for prime $N$ found in [7, Theorem 2].

We see that calculation of the bound on $R\left(Q_{m}\right)$ given in (3.5) requires the evaluation of four sums. To obtain a bound that is easier to calculate, we can make use of (3.7) and the corresponding lower bound

$$
S_{k}>2 \log (k)+\beta-\frac{4}{k^{2}},
$$

which may also be derived from [7, Lemmas 1 and 2]. Then we have the following corollary. 
Corollary 5. For $1 \leq m \leq s, n \geq 2$, and $r$ a prime number so that $r$ does not divide $n$, there exists a $\mathbf{z} \in Z_{r}$ such that

$$
\begin{aligned}
R\left(Q_{m}\right)< & \frac{1}{r}\left(1+\frac{2 \log \left(n^{m-1} r\right)+\beta+\frac{1}{n^{2 m-2} r^{2}}}{n}\right)^{m} \\
& \times\left(1+2 \log \left(n^{m} r\right)+\beta+\frac{1}{n^{2 m} r^{2}}\right)^{s-m} \\
& +\frac{r-1}{r}\left(1-\frac{2 \log (r)-\frac{4+r^{2}}{n^{2 m-2} r^{2}}}{n(r-1)}\right)^{m}\left(1-\frac{2 \log (r)-\frac{4+r^{2}}{n^{2 m} r^{2}}}{r-1}\right)^{s-m}-1
\end{aligned}
$$

\section{Numerical Results}

Here we give the results of some calculations of $R\left(Q_{m}\right)$ with $s=7$ that were obtained by making use of Theorem 2 and (2.5). The required functions $F_{N}$ and $F_{N / n}$ were calculated using the method given in [5]. The actual lattice rules $Q_{m}$ used were taken from [3]. There, $n$ was chosen to be 2, and for each $m$ and $r$, the integer vectors $\mathbf{z}$ required in (1.8) were of the one-parameter Korobov form

$$
\mathbf{z}(a)=\left(1, a, a^{2}, \ldots, a^{6}\right) \quad(\bmod r), \quad 1 \leq a<r .
$$

For each $m$ and $r$, the value of $a$ used was obtained by minimizing the quantity $P_{2}\left(Q_{m}\right)$. For a lattice rule $Q$, this quantity is defined by

$$
P_{2}=P_{2}(Q):=\sum_{\mathbf{h} \in L^{\perp}}^{\prime} \frac{1}{\left(\bar{h}_{1} \bar{h}_{2} \cdots \bar{h}_{s}\right)^{2}},
$$

and is another widely-used criterion for measuring the goodness of lattice rules (see, for example, $[1,3,4,8,11]$ ).

Values of $R\left(Q_{m}\right)$ for lattice rules of approximate order 15000,18000 , and 24000 are given in Tables 1, 2, and 3 (next page) respectively. Most of the lattice rules taken from [3] did not have $r$ prime, and, in fact, of the 24 values of $r$ used, only six were prime. For these six values, the bounds on $R\left(Q_{m}\right)$ given by $M_{m, r}^{(n)}$ are also listed in the tables. As can be seen, the bounds are quite reasonable.

The expression for $M_{m, r}^{(n)}$ given on the right-hand side of (3.5) indicates that the largest contribution to $M_{m, r}^{(n)}$ would come from $H_{m, r}^{(n)}$, where

$$
H_{m, r}^{(n)}=\frac{1}{r}\left(1+\frac{S_{n^{m-1} r}}{n}\right)^{m}\left(1+S_{n^{m} r}\right)^{s-m} .
$$

The proof of Theorem 3 shows that $H_{m, r}^{(n)}$ is just $f_{N}^{(n)}(\mathbf{0}) / r$. Thus, this quantity will always be present in an expression for $M_{m, r}^{(n)}$ regardless of whether $r$ is prime or not. The values of this quantity (with $n=2$ ) are given in the tables, and they appear to provide a useful bound on $R\left(Q_{m}\right)$. Certainly for $r$ prime, 
TABLE 1

\begin{tabular}{cccccc}
\hline$m$ & $r$ & $N=2^{m} r$ & $R$ & $M_{m, r}^{(2)}$ & $H_{m, r}^{(2)}$ \\
0 & 15019 & 15019 & 85292.13 & & 85293.40 \\
1 & 7509 & 15018 & 83642.07 & & 83647.83 \\
2 & 3753 & 15012 & 82040.46 & & 82042.08 \\
3 & 1877 & 15016 & 80450.10 & 80451.22 & 80451.26 \\
4 & 937 & 14992 & 78933.33 & 78934.32 & 78934.39 \\
5 & 469 & 15008 & 77384.85 & & 77385.74 \\
6 & 233 & 14912 & 76030.79 & 76031.32 & 76031.50 \\
7 & 117 & 14976 & 74470.21 & & 74470.84 \\
\hline
\end{tabular}

TABLE 2

\begin{tabular}{cccccc}
\hline$m$ & $r$ & $N=2^{m} r$ & $R$ & $M_{m, r}^{(2)}$ & $H_{m, r}^{(2)}$ \\
0 & 18101 & 18101 & 80549.58 & & 80550.38 \\
1 & 9049 & 18098 & 79025.61 & 79027.31 & 79027.32 \\
2 & 4525 & 18100 & 77525.14 & & 77526.39 \\
3 & 2261 & 18088 & 76070.72 & & 76072.08 \\
4 & 1131 & 18096 & 74618.91 & & 74619.68 \\
5 & 565 & 18080 & 73223.85 & & 73224.60 \\
6 & 281 & 17984 & 71950.82 & 71951.35 & 71951.50 \\
7 & 141 & 18048 & 70510.86 & & 70511.52 \\
\hline
\end{tabular}

TABLE 3

\begin{tabular}{cccccc}
\hline$m$ & $r$ & $N=2^{m} r$ & $R$ & $M_{m, r}^{(2)}$ & $H_{m, r}^{(2)}$ \\
0 & 24041 & 24041 & 73508.53 & & 73509.63 \\
1 & 12019 & 24038 & 72155.21 & & 72156.72 \\
2 & 6009 & 24036 & 70826.53 & & 70827.72 \\
3 & 3005 & 24040 & 69516.33 & & 69517.51 \\
4 & 1501 & 24016 & 68256.59 & & 68257.37 \\
5 & 751 & 24032 & 66983.13 & 66983.78 & 66983.86 \\
6 & 375 & 24000 & 65775.73 & & 65776.40 \\
7 & 187 & 23936 & 64617.34 & & 64617.94 \\
\hline
\end{tabular}

the results in the tables show that numerically there is very little difference between $M_{m, r}^{(n)}$ and $H_{m, r}^{(n)}$. For fixed $n$ and fixed $N=n^{m} r$, we would expect $H_{m, r}^{(n)}$ to decrease as $m$ increased, and this is what is observed in each of the three tables where the values of $N$ are approximately the same. This suggests that for lattice rules of the form (1.8) with approximately the same order, one might expect the rules of higher rank to have smaller values of $R$ than the rules of lower rank. 


\section{BIBLIOGRAPHY}

1. S. A. R. Disney and I.H. Sloan, Lattice integration rules of maximal rank formed by copying rules of Korobov type, SIAM J. Numer. Anal. 29 (1992), 566-577.

2. E. Hlawka, Zur angenäherten Berechnung mehrfacher Integrale, Monatsh. Math. 66 (1962), 140-151.

3. S. Joe and S. A. R. Disney, Intermediate rank lattice rules for multidimensional integration, SIAM J. Numer. Anal. 30 (1993), 569-582.

4. S. Joe and I. H. Sloan, Imbedded lattice rules for multidimensional integration, SIAM J. Numer. Anal. 29 (1992), 1119-1135.

5.

6. N. M. Korobov, The approximate computation of multiple integrals, Dokl. Akad. Nauk SSSR 124 (1959), 1207-1210. (Russian)

7. H. Niederreiter, Existence of good lattice points in the sense of Hlawka, Monatsh. Math. 86 (1978), 203-219.

8. $\ldots$, The existence of efficient lattice rules for multidimensional numerical integration, Math. Comp. 58 (1992), 305-314, S7-S16.

9. I. H. Sloan and P. J. Kachoyan, Lattice methods for multiple integration: theory, error analysis and examples, SIAM J. Numer. Anal. 24 (1987), 116-128.

10. I. H. Sloan and J. N. Lyness, The representation of lattice quadrature rules as multiple sums, Math. Comp. 52 (1989), 81-94.

11. I.H. Sloan and L. Walsh, $A$ computer search of rank-2 lattice rules for multidimensional quadrature, Math. Comp. 54 (1990), 281-302.

School of Mathematics, University of New South Wales, Sydney, New South Wales 2033, Australia

Current address: Department of Mathematics \& Statistics, The University of Waikato, Private Bag 3105, Hamilton, New Zealand

E-mail address: stephenj@waikato.ac.nz 